03

\title{
Анизотропия ИК поглощения сверхтонкого слоя воды на межфазной границе вода/кварцевое стекло
}

\author{
(C) В.М. Золотарев \\ Университет ИТМО, \\ 197101 Санкт-Петербург, Россия \\ e-mail: VM-Zolotarev@mail.ru
}

Поступила в редакцию 16.04.2019 г.

В окончательной редакции 16.04.2019 г.

Принята к публикации 16.05.2019 г.

Методом НПВО исследованы оптические свойства сверхтонкого слоя воды в зоне контакта двух диэлектриков $(\mathrm{ZnSe}-\mathrm{SiO} 2)$. Получены эффективные значения показателей преломления и поглощения в области полосы валентных колебаний группы ОН. Обнаружена плоскостная ориентация молекул воды на границе межфазного слоя вода/кварцевое стекло.

Ключевые слова: НПВО, валентные колебания, ион гидроксония, жидкая вода, лед.

DOI: $10.21883 /$ OS.2019.12.48687.142-19

Свойства воды на межфазных границах активно исследуются разными физическими методами, поскольку эти свойства играют важную роль в химических процессах, протекающих в тропосфере с участием частиц аэрозоля, покрытого тонким слоем молекулярной воды [13]. Такие реакции зависят от концентрации водяного пара, поскольку относительная влажность определяет равновесную концентрацию воды на поверхности [3]. Кроме того, изучение свойств воды на межфазных границах важно для понимания химических процессов, протекающих на поверхности, для целого ряда направлений техники, включая оптические технологии [4-7], а также для геологии, биологии и экологии [8-11]. Для исследования межфазных границ воды с твердой фазой, например в системах $\mathrm{H}_{2} \mathrm{O}-\mathrm{SiO}_{2}, \mathrm{H}_{2} \mathrm{O}-\mathrm{SiO}_{2}-\mathrm{Si}$, $\mathrm{H}_{2} \mathrm{O}-\mathrm{Ag}$, применяется целый ряд физических методов, включая рентген [12] и комбинационное рассеяние в режиме SERS (surface-enhanced Raman spectroscopy) [13]. Однако наиболее широко используются традиционные методы поляризационной ИК спектроскопии неполного внутреннннего отражения (НПВО) [14], а также метод сумма-частотной колебательной спектроскопии (sumfrequency vibrational spectroscopy (SFVS)) [15-17]. Эти исследования связаны с изучением валентных колебаний ОН-групп или комбинированных частот, где используется метод SFVS-спектроскопии [17]. В большинстве этих работ анализируются спектроскопические параметры (частота, интенсивность, полуширина) ИК полосы и не всегда учитывается другая оптическая характеристика межфазной границы, а именно показатель преломления, значение которого зависит от физико-химических условий существования граничного слоя. В связи с этим можно отметить, что увеличение толщины слоя воды будет минимизировать эффекты поверхности (например, влияние ее микрорельефа на показатель преломления слоя). В работе [14] отмечается влияние кислотности границы раздела вода-кварц на спектроскопические параметры как основной полосы валентных колебаний воды $v_{\mathrm{OH}}$, так и на комбинированную частоту $\left(v_{\mathrm{comb}}=v_{\mathrm{OH}}+\delta_{\mathrm{HOH}}\right)$. Спектроскопические эффекты в работах интерпретируются как „синее“ или „красное“ смещение или как образование „жидкой“ или ,льдоподобной“ фазы воды [12-18]. Так, например, в работе [14] изучалась усредненная ориентация молекул воды на границе с $\mathrm{SiO}_{2}$ путем измерения дихроичного отношения $s$ - и $p$ поляризованных компонент в спектре НПВО. Показано, что при низкой относительной влажности образуется льдоподобная структура с сильной водородной связью, в результате получается дихроичное отношение, равное примерно 0.4, тогда как для жидкой воды дихроичное отношение близко к 1.0. При увеличении относительной влажности дихроичное отношение обеих структур воды приближается к величине $0.7-0.8$, что согласуется с ориентацией молекул, типичной для объемной воды [14].

В свою очередь, независимые диэлектрические измерения слоев воды толщиной 10-200 А̊ показывают, что для толщин слоев в 2-3 молекулы наблюдается сильное уменьшение диэлектрической проницаемости $\varepsilon(\varepsilon=2)$ по сравнению с жидкой водой $(\varepsilon=80)$ [19], что связано с уменьшением подвижности молекул вблизи границы раздела вода- $\mathrm{SiO}_{2}$. В этой работе [19] отмечается, что „несмотря на обширные исследования, диэлектрическая проницаемость поверхностной воды и ее глубина остаются по существу неизвестными, потому что измерения сложны“. Подобные трудности существуют также и при изучении сверхтонких пленок воды методами ИК спектроскопии по причине измерения малых сигналов и разнообразию факторов (гетерогенность поверхности и, как следствие, неоднородность покрытия поверхности стекла водяной пленкой при низкой относительной 
влажности и др.), влияющих на результаты измерений $[1,7,14]$.

Цель настоящей работы - получение данных об оптических свойствах сверхтонкого слоя воды в области валентных колебаний группы ОН на границе раздела вода-кварцевое стекло.

\section{Методика подготовки образцов и техника измерений}

Сверхтонкие слои воды были получены путем соединения двух плоских поверхностей полированных пластин, изготовленных из кварцевого стекла и кристалла $\mathrm{ZnSe}$, которые были соединены с помощью оптического контакта. Особенности подготовки и методы очистки поверхностей контактной пары из разнородных материалов рассмотрены в работах [20-22]. Такой способ соединения двух полированных пластин позволяет получить в зазоре между пластинами сверхтонкий слой воды, который по оценкам разных авторов находится в пределах 100-1000 $[23-28]$.

Пластина из кристалла ZnSe была приготовлена в виде элемента НПВО на 6 отражений для угла $45^{\circ}$, что позволяло проводить исследования адсорбированного слоя воды, находящегося между пластиной из кварцевого стекла и кристаллом ZnSe. Такой прием позволил за счет увеличения числа отражений повысить чувствительность при измерениях сверхтонких слоев воды и достигнуть лучшего усреднения результатов. Последнее качество эксперимента связано с большой площадью проекции пучка излучения на границе раздела, который распространяется внутри элемента НПВО при скользящих углах падения около $73^{\circ}$. Промывка пластин перед их соединением осуществлялась в деионизованной воде, что, однако, не избавляло полностью от наличия углеводородов на поверхности пластин. Инфракрасные спектры НПВО в диапазоне 5000-600 $\mathrm{cm}^{-1}$ были получены на ИК фурье-спектрометре TENSOR 27 фирмы Bruker с разрешением $2 \mathrm{~cm}^{-1}$ для 1000 сканов с использованием поляризованного излучения. Конфигурация элемента НПВО позволяла использовать его для получения наклонного угла падения $73^{\circ}$ при числе отражений 2 (рис. 1). Для получения поляризованного излучения использовались дифракционные поляризаторы 1200 и 600 lines/mm на подложках: кристалл $\mathrm{CaF}_{2}$, стекло ИКС-35.

\section{Методика эксперимента}

Присутствие углеводородов на поверхности соединяемых пластин использовалось в качестве внутреннего стандарта для оценки эффективной толщины пленки в контактном слое между пластинами. Данные об оптических параметрах (показатели преломления, поглощения) типичных углеводородов (нефтепродукты), присутствующих в виде аэрозоля в атмосфере и, в частности, в

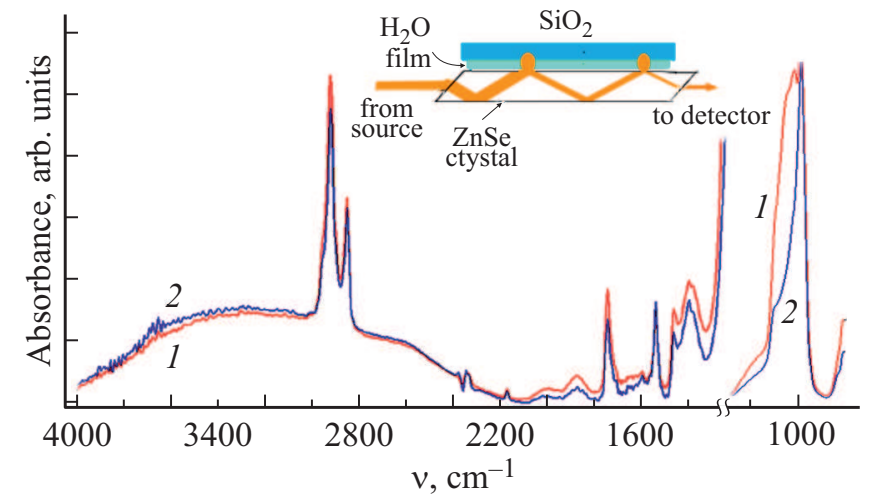

Рис. 1. Спектр НПВО (эксперимент, система $\mathrm{ZnSe}\left(n_{1}\right)-$ пленка $\mathrm{H}_{2} \mathrm{O}\left(n_{2}\right)-\mathrm{SiO}_{2}\left(n_{3}\right)$, угол $\left.\theta=73^{\circ}\right): 1-p$-поляризация, $2-$ $s$-поляризация. Участок $4000-1400 \mathrm{~cm}^{-1}$ дан с увеличением масштаба $50^{+}$по ординате. В спектре наряду с водой присутствуют углеводороды, интенсивная полоса $2922 \mathrm{~cm}^{-1}$. $\mathrm{B}$ верхней части рисунка показана схема эксперимента для угла $73^{\circ}$.

производственных помещениях, в которых производится изготовление и сборка оптических деталей, были получены из работ [29-31]. Для изучения структурных свойств контактного слоя использовались соотношения, выполняющиеся для тонкой изотропной пленки для компонент $R_{s}$ и $R_{p}$ отраженного поляризованного излучения [32,33].

В условиях НПВО для малых значений коэффициента поглощения $K$

$$
R=\exp (-K d e) \approx(1-A),
$$

где $A-$ ослабление пучка излучения, $K=4 \pi k / \lambda\left[\mathrm{cm}^{-1}\right]-$ коэффициент поглощения, $k-$ мнимая часть при комплексном показателе преломления $\overline{\bar{n}}=n(1-i k), \lambda-$ длина волны, de - эффективная толщина поглощающего слоя,

$$
d e=\frac{n_{21} E_{0}^{2} d}{\cos \theta}
$$

где $n_{21}=n_{2} / n_{1}, d-$ геометрическая толщина пленки, $\theta-$ угол падения, $E_{0}-$ амплитуда напряженности электрического поля вблизи границы раздела, зависящая от $s$ - или $p$-поляризации.

Для тонкой изотропной пленки с учетом системы $\mathrm{ZnSe}\left(n_{1}\right)$-пленка $\left(n_{2}\right)-\mathrm{SiO}_{2}\left(n_{3}\right)$ [32]:

$$
A_{s}=1-R_{s}=K d e_{s}=\frac{4 n_{21} d \cos \theta}{\left(1-n_{31}^{2}\right)},
$$

$A_{p}=1-R_{p}=K d e_{p}=\frac{4 n_{21} d \cos \theta\left[\left(1+n_{32}^{4}\right) \sin ^{2} \theta-n_{51}^{2}\right]}{\left(1-n_{31}^{2}\right)\left[\left(1+n_{31}^{2}\right) \sin ^{2} \theta-n_{31}^{2}\right]}$

где $n_{21}=n_{2} / n_{1}, n_{31}=n_{3} / n_{1}, n_{32}=n_{3} / n_{2}$ - относительные показатели преломления. Определив из эксперимента отношение $A_{p} / A_{s}$, можно получить значение $n_{2}-$ показателя преломления пленки, т.е. тонкого изотропного слоя, находящегося между пластинками $\mathrm{ZnSe}-\mathrm{SiO}_{2}$. 
Если объект анизотропен, то следует учитывать особенности конфигурации поля $E$ для условий НПВО. Поле $E$ в средах $n_{2}$ и $n_{3}$ существует в трех ортогональных направлениях $x y z$. Условия эксперимента выбраны таким образом, что на границе раздела сред компонента $E_{x}$ перпендикулярна плоскости падения, а компоненты $E_{y}$ и $E_{z}$ лежат в плоскости падения, при этом $E_{z}$ перпендикулярна отражающей поверхности элемента НПВО.

Коэффициенты анизотропии поглощения тонкой пленки $k_{x}, k_{y}, k_{z}$ связаны с параметрами $A$ соотношениями $A_{s}=\alpha k_{x}, A_{p}=\beta k_{y}+\gamma k_{z}$. Величины $\alpha, \beta, \gamma$ зависят от компонент напряженности электрического поля $E_{x}^{2}, E_{y}^{2}$, $E_{z}^{2}$, которые в свою очередь зависят от $n_{21}, n_{31}, n_{32}$, угла $\theta$ и толщины пленки $d$. При таком подходе с учетом малости значений $k_{x}, k_{y}, k_{z}$ традиционно исходят из допущения, что для пленки $n_{x} \approx n_{y} \approx n_{z}$, при этом аналитические выражения для $\alpha, \beta, \gamma$ имеются только для случая полубесконечной среды $n_{2}$ [34].

\section{Результаты эксперимента}

Анализ полученных спектров (рис. 1) показывает, что полоса $3400 \mathrm{~cm}^{-1}$ в спектре НПВО валентных колебаний тонкого слоя воды намного шире по сравнению с соответствующей полосой жидкой воды. На контуре полосы в спектре НПВО можно выделить максимумы $3640,3380,3200-3000,2670 \mathrm{~cm}^{-1}$. Видны также типичные полосы 2922, 1460, 1398, $1742 \mathrm{~cm}^{-1}$ углеводородов, адсорбированных на поверхности. Полосы с максимумами в окрестности $3640,3380,3200-3000 \mathrm{~cm}^{-1}$ относят к полосам с разорванными Н-связями и с Нсвязями, типичными для жидкой воды и льда соответственно [14,35-37]. Полоса $2670 \mathrm{~cm}^{-1}$, обнаруженная в нашем эксперименте, может быть отнесена к валентным колебаниям иона гидроксония $\mathrm{H}_{3} \mathrm{O}+$ [38]. Появление полос гидроксония в спектре НПВО обусловлено взаимодействием молекул воды с поверхностью кварцевого стекла, которое проявляет кислотные свойства, что связано с образованием в поверхностном слое по схеме $\left(\mathrm{SiO}_{2}\right)_{x}+2 \mathrm{H}_{2} \mathrm{O} \rightleftarrows \mathrm{H}_{2} \mathrm{SiO}_{4}+\left(\mathrm{SiO}_{2}\right)_{x}-1$ очень слабой, малорастворимой в воде кислоты с формулой $n \mathrm{SiO}_{2} \cdot m \mathrm{H}_{2} \mathrm{O}$. Вероятность образования такого соединения весьма высока, если принять во внимание, что система $\mathrm{ZnSe}-\mathrm{SiO}_{2}$ находилась в длительном контакте (около 30 лет).

Из рис. 1 можно заметить качественное согласие между данными эксперимента и расчета для $A_{s}$ - и $A_{p}$ компонент в случае полос углеводородов у 2922, 1460 , $1398 \mathrm{~cm}^{-1}$, а также для обертонов $2002,1876 \mathrm{~cm}^{-1}$ и основных полос 1100 и $860 \mathrm{~cm}^{-1}$ кварцевого стекла. Вместе с тем для полосы $3400 \mathrm{~cm}^{-1}$ воды и узкой полосы $1540 \mathrm{~cm}^{-1}$ (возможно, это полоса карбоксилатаниона) соотношение компонент $A_{s}$ и $A_{p}$ не соответствует расчетным данным (рис. 2), полученным в предположении изотропного распределения молекул в толще слоя между пластинами $\mathrm{ZnSe}-\mathrm{SiO}_{2}$.

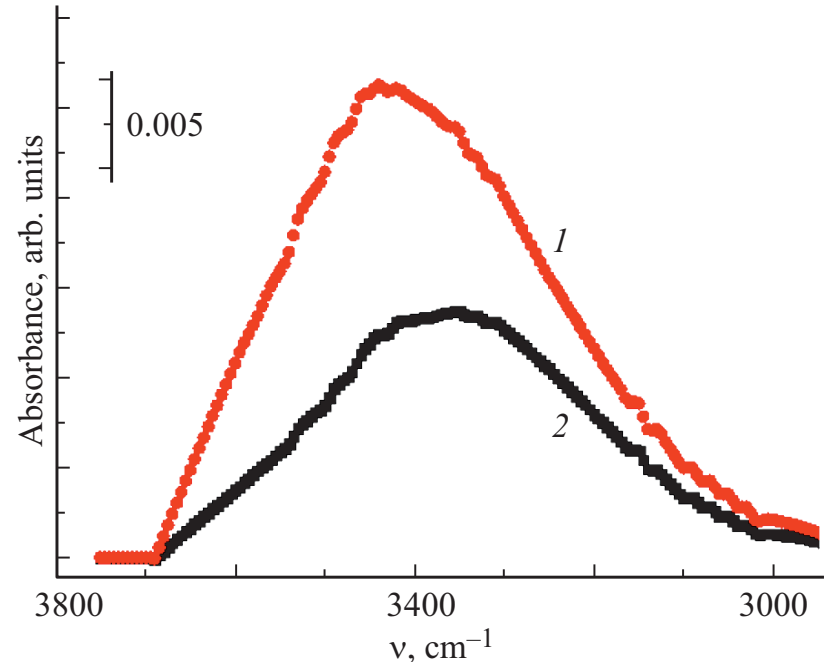

Рис. 2. Спектр НПВО тонкой пленки $d=100 \AA$ жидкой воды (расчет, система $\mathrm{ZnSe}\left(n_{1}\right)$-пленка $\mathrm{H}_{2} \mathrm{O}\left(n_{2}\right)-\mathrm{SiO}_{2}\left(n_{3}\right)$, угол $\left.\theta=73^{\circ}\right): 1-p$-поляризация, $2-s$-поляризация.

В связи с этим для полосы $2922 \mathrm{~cm}^{-1}$ отношение $A_{p} / A_{s}$ было использовано для определения $n_{2}-$ показателя преломления пленки адсорбированных углеводородов, который оказался равным $1.65 \pm 0.05$. Для сравнения типичное значение $n$ для моторных масел равно 1.46 [28]. Превышение полученного значения эффективного показателя преломления для пленки углеводородов в контактном слое между $\mathrm{ZnSe}-\mathrm{SiO}_{2}$ по отношению к типичному значению $n$ для жидких углеводородов (моторное масло) связано с влиянием на результаты измерений микрошероховатостей контактирующих поверхностей (наибольший вклад вносят микрошероховатости кристалла $\mathrm{ZnSe}$ ). Данные независимого метода эллипсометрии для контактной пары $\mathrm{SiO}_{2}-\mathrm{SiO}_{2}$ также показывают на завышенное значение $n_{2}=1.41-1.43$ для зоны контактного слоя, в котором находится вода [26]. Влияние микрошероховатостей на эффективный показатель преломления воды в контактном слое рассмотрен в работе [23].

Эффективная толщина пленки углеводородов $d$ в контактном слое между $\mathrm{ZnSe}-\mathrm{SiO}_{2}$ составила 80-100 $\AA$. Оценка выполнена с помощью уравнения (3) на основе значений коэффициента поглощения $K$ углеводородов [28-30]. Данные для толщины пограничного слоя, полученные для контактной пары $\mathrm{SiO}_{2}$-пленка $\mathrm{H}_{2} \mathrm{O}-\mathrm{SiO}_{2}$ методом эллипсометрии, дают оценку $d=30-60 \AA$ [27]. Оценки разных авторов для эффективной толщины зоны контактного слоя находится в пределах 100-1000 $\AA$ [2328]. Исходя из оценки величины микрошероховатостей соединяемых поверхностей считается, что наиболее вероятная толщина зоны контактного слоя находится в интервале 0-200 $\AA$ [23-25].

Малое отличие экспериментальных значений $A_{s}$ и $A_{p}$ для полосы $3400 \mathrm{~cm}^{-1}$ (рис. 1) по сравнению с расчетом для изотропного слоя воды (рис. 2) указыва- 
ет на анизотропию поглощения в приграничном слое вода- $\mathrm{SiO}_{2}$. Используя полученное значение $d=100 \AA$ и $n_{2}=1.65 \pm 0.05$ для углеводородов, можно, определив $R_{s}$ для полосы $3400 \mathrm{~cm}^{-1}$, вычислить с помощью (3) показатель поглощения $k_{x}$ для эффективного слоя воды в зоне контакта. Вычисленный таким путем показатель поглощения воды $k_{x}=0.33 \pm 0.06$, что близко к значению $k=0.289$ для жидкой воды [29]. Вычисленное значение $k_{x}$ определяет поглощение диполей молекул воды вдоль оси $X$, соответственно такое же значение $k_{y}$ в силу симметрии будет и для оси $Y$. Соотношения между величинами $\alpha, \beta, \gamma$, необходимые для определения $k_{z}$, можно оценить в приближении $d \ll \lambda$, исходя из того, что для сверхтонкой пленки значения величин $E_{x}^{2}, E_{y}^{2}, E_{z}^{2}$ будут близки для случая $d \rightarrow 0$ [33]. Для конкретного случая $n_{1}=2.4, n_{3}=1.45$ и угла $\theta=73^{\circ}$ величины $E_{x}^{2}, E_{y}^{2}, E_{z}^{2}$ равны $0.757,0.570,0.775$ соответственно [33]. Таким образом, значение параметра $\gamma$ будет превышать величину $\beta$. Поэтому для объяснения уменьшения величины $R_{s}$, полученной из эксперимента для полосы валентных колебаний воды у $3400 \mathrm{~cm}^{-1}$ с учетом того, что $k_{x}=k_{y}$, следует, что $k_{z}<k_{y}$ и соответственно должна быть меньше $k_{x}$. Такой подход позволяет оценить величину $k_{z}$, которая оказывается равной $0.160 \pm 0.05$.

Независимо значение $k_{z}$ можно оценить из соотношения $k=\left(k_{x}+k_{y}+k_{z}\right) / 3$, допуская, что среднее значение показателя поглощения $k$ на границе вода- $\mathrm{SiO}_{2}$ близко к величине поглощения жидкой воды. Вычисленная таким способом величина $k_{z}=0.21 \pm 0.05$ является эффективной и характеризует всю толщу пленки. В действительности, показатель $k_{z}$ должен быть меньше этой оценки, поскольку толщина слоя воды $100 \AA \AA$, найденная из эксперимента, заметно превышает количество молекулярных слоев воды, на которые распространяется воздействие сил поверхности $[14,20]$. Оценка толщины слоя воды с помощью (3), полученная на основе вычисленного значения $k_{x}$ в полосе $3400 \mathrm{~cm}^{-1}$ для системы $\mathrm{ZnSe}$-пленка $\mathrm{H}_{2} \mathrm{O}-\mathrm{SiO}_{2}$ составила $d=42 \pm 16 \AA$, что согласуется с ранее выполненными оценками [23-28]. Следует отметить, что учет интегрального поглощения интенсивности полосы $3400 \mathrm{~cm}^{-1}$ увеличивает вычисленное значение $d$ пленки воды в зоне контакта примерно в два раза.

В заключение можно отметить, что выполненные с помощью метода НПВО поляризационные спектроскопические исследования оптических свойств молекулярной воды, граничащей с поверхностью $\mathrm{SiO}_{2}$, позволили выполнить численные оценки коэффициентов анизотропии поглощения для валентной полосы $3400 \mathrm{~cm}^{-1}$ сверхтонкого слоя воды. Полученные данные указывают на плоскостную ориентацию молекул воды на межфазной границе вода- $\mathrm{SiO}_{2}$, что согласуется с данными, полученными независимым методом диэлектрических измерений.

\section{Список литературы}

[1] Sumner A.L., Menke E.J., Dubowski Y. et al. // Phys. Chem. 2004. V. 6. P. 604-613.

[2] Svensson R., Ljungstrom E., Lindqvist O. // Atm. Environ. 1987. V. 21. P. 1529.

[3] Bjorneholm O., Hansen M.H., Hodgson A. et al. //Chem. Rev. 2016. V. 116. P. 7698-7726.

[4] Первеев А.Ф., Золотарев В.М., Егоров П.П., Муранова Г.А. // Опт. и спектр. 1972. Т. 32. С. 607-611.

[5] Ashrit P.V., Badilescu S., Girouard F.E., Vo-Van Truong // Appl. Opt. 1989. V. 28. P. 420-422. doi 10.1364/AO.28.000420

[6] Золотарев В.М., Первеев А.Ф. // ДАН СССР. 1972. Т. 204. C. 903-905.

[7] Poda A. Diss. PhD. Interfacial Engineering of MicroStructured Materials. Auburn. Alabama, 2010.

[8] Yeşilbaş M. Diss. PhD. Thin Water and Ice Films on Minerals: A Molecular Level Study. Umeøa, Sweden, 2018.

[9] Du Q., Freysz E., Shen Y.R. // Phys. Rev. Lett. 1994. V. 72. P. 238-241.

[10] Takeuchi M., Martra G., Coluccia S., Anpo M. // J. Near Infrared Spectrosc. 2009. V. 17. P. 373-384.

[11] Trung Quan Luong. Diss. PhD. Terahertz and Infrared Spectroscopy of Confined Water. Ruhr University. Bochum, 2012.

[12] Verdaguer A., Weis C., Oncins G., Ketteler G., Bluhm H., Salmeron M. [Электронный ресурс ]. Режим доступа: https://escholarship.org/uc/item/3sw5003d

[13] Shin D., Hwang J., Jhe W. // Nature Commun. 2019. V. 10. P. 286. doi 10.1038/s41467-019-08292-0

[14] Barnette A.L., Asaya D.B., Kim S.H. // Phys. Chem. Chem. Phys. 2008. V. 10. P. 4676-4677.

[15] Shen Y.R. // Frontier in Laser Spectroscopy/ Ed. by Hansch T.W., Inguscio M. P. 139-165. Amsterdam: North Holland, 1994.

[16] Ostroverkhov V., Waychunas G.A., Shen Y.R. // Phys. Rev. Lett. 2005. V. 94. P. 046102.

[17] Isaienko O., Nihonyanagi S., Sil D., Borguetdx E. // J. Phys. Chem. Lett. 2013. V. 4. P. 531-535. doi org/10.1021/jz3015088

[18] Yang J., Wang E.G. // Phys. Rev. B. 2006. V. 73. P. 035406

[19] Fumagalli L., Ferrari G., Sampietro M., Gomila G. // Appl. Phys. Lett. 2007. V. 91. P. 243110.

[20] Fumagalli L., Esfandiar A., Fabregas $R$. et al. // Science. 2018. V. 360. P. 1339-1342. doi 10.1126/science.aat4191

[21] Милехин A.Г., Himcinschi C., Friedrich M. et al. // ФТП. 2006. T. 40. N 11. C. $1338-1347$.

[22] Обреимов И.В., Трехов Е.С. // ЖЭТФ. 1957. Т. 32. С. 185.

[23] Золотарев В.М. // Физическая энциклопедия. Т. 3. С. 302. М.: „Советская энциклопедия“. Гл. ред. Прохоров А.М. 1988.

[24] Holt R.B. Research on optical contact bonding. Paperback, 1966.

[25] Haisma J., Spierings G.A. // Mater. Sci. Engineer. V. 37. P. 1-60. doi 10.1016/s0927-796x (02)00003-7

[26] Качкин С.С. // Оптический журнал. 1997. № 7. С. 51-53.

[27] Елхин П.М., Лисищын Ю.В., Поздняков О.Ф., Юдин В.С. // ФХС. 1986. № 5. С. 611-614.

[28] Абаев М.И., Лисицын Ю.В., Путилин Э.С. // Письма в ЖТФ. 1984. Т. 4. С. $1505-1507$. 
[29] Золотарев В.М., Морозов В.Н., Смирнова Е.В. Оптические постоянные природных и технических сред. Л.: Химия, 1984. 216 с.

[30] McGinty S., Kapala M., Niedziela R. // Phys. Chem. Chem. Phys. 2009. P. 7998-8004. doi 10.1039/b905371a

[31] Roman B. Dissertation. Mid-Infrared Complex Refractive Indices for Motor Oil. DePaul University. Chicago IL, 2017. doi org/10.1002/essoar.10500283.1

[32] Харрик Н. Спектроскопия внутреннего отражения. М.: Мир, 1970. 335 c.

[33] Mirabella F.M., Harrick N. J. Internal Reflection Spectroscopy: Review and Supplement. N.Y.: Harrick Sci. Corp., 1985, $195 \mathrm{p}$.

[34] Flournoy P.A., Schaffers W.J. // Spectrochim. Acta. 1966. V. 22. P. 5.

[35] Kühne T. D., Pascal T.A., Kaxiras E., Jung Y. // J. Phys. Chem. Lett. 2010. V. 2. P. 105-113.

[36] Zhang C., Khaliullin R.Z., Bovi D., Guidoni L., Kühne T.D. // J. Phys. Chem. Lett. 2013. V. 4. P. 3245-3250.

[37] Totun B., Kunze C., Zhang C. et al. // Phys. Chem. Chem. Phys. V. 2014. P. 7377-7384.

[38] Юхневич Г.В. Инфракрасная спектроскопия воды. М.: Наука, 1973. 208 с. 